\title{
Experimental Verification of the Fire Dynamics Simulator Hydrodynamic Model
}

\author{
J M CLEMENT, \\ Holmes Fire \& Safety Ltd, \\ Sydney, Australia \\ jason@holmesfire.com
}

\author{
C M FLEISCHMANN \\ University of Canterbury, \\ Christchurch, New Zealand \\ charley@civil.canterbury.ac.nz
}

\begin{abstract}
This paper summarises a series of salt water experiments that resemble the smoke flow in the early stages of a residential scale building fire. Experimental measurements of the fluid density of the salt water flow field are made using the Laser Induced dye Fluorescence (LIF) technique. The salt water experiments are then simulated using the

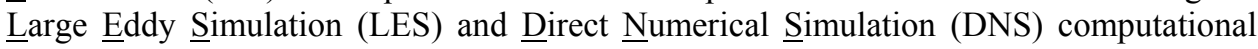

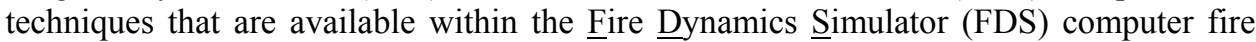
model. Computations, using each technique, are conducted at a number of different grid resolutions. The comparison of computational results and experimental measurements show that the LES technique performs better at coarse grid resolutions, due primarily to the computational stability requirement for an artificially high fluid viscosity in DNS, which inhibits turbulent mixing. The LES computations were capable of resolving the flow field features that are evident in the experimental density measurements when the uniform grid resolution has a characteristic dimension that is less than the vertical extent of the domain divided by fifty. Generally speaking, at high grid resolutions, the agreement between experimental measurements and the LES computational results were very good.
\end{abstract}

KEYWORDS: Computational Fluid Dynamics, FDS, DNS, LES, Salt water Modelling

\section{INTRODUCTION}

At the present time fire modelling is going through a stage of rapid development. The increasing computational power is making field modelling a progressively more feasible option to use in fire research and design. At the forefront of recent fire model development is the Fire Dynamics $\underline{S}$ imulator $(\mathrm{FDS})^{[1]}$. The recent release of this model into the public domain, coupled with the promising results that have been seen in Large

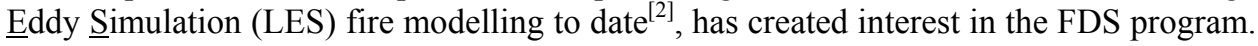
Of particular interest to potential users and fire safety design authorities is the accuracy of the computational results.

Unfortunately, it is beyond our capabilities to determine a complete analytical solution for turbulent fire induced smoke flow. In order to assess the accuracy of computational fire modelling we are forced to compare the results from computational simulations with experimental measurements from a physical simulation. When conducting computational simulations of fire experiments it is necessary to invoke several sub-models that account for heat transfer and combustion processes, which influence the thermal gas flow. In the subsequent comparison of these computational results with experimental measurements it 
is difficult to know whether or not the performance of any specific sub-model is responsible for inaccuracies in the computational simulations. Most likely it is a combination of the performance of all the sub-models employed. Without knowledge of how the inaccuracies are proportioned between the sub-models, there is uncertainty on where improvement efforts should be focussed in the progressive development of the modelling approach. Thus to assess the accuracy of a computational fire model, it is desirable to assess the performance of each sub-model independently. The hydrodynamic model is a fundamental component of a field model that approximates the motion and the properties of the fluid from the physical forces that are present in the system. In the progressive assessment of the FDS model accuracy, determining the performance of this fundamental component is the most logical place to start.

In this research the aim is to conduct a specific assessment of the accuracy of the hydrodynamic model within the FDS software, without applying other sub-models that are used to simulate fire related phenomenon. The accuracy of the hydrodynamic model will be assessed as a function of the two user specified computational techniques i.e.

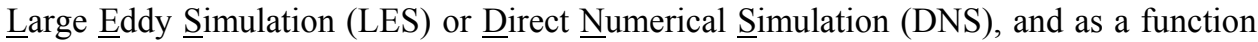
of grid resolution. To achieve this aim, the flow to be simulated must be buoyant, inert and conserve all the buoyancy that is released at the source. As such, a series of buoyant salt water experiments have been conducted to generate suitable experimental data, pertinent to fire induced smoke flow, for comparison with the computational predictions. Previous fire research ${ }^{[3][4][5]}$ has employed salt water modelling as a means of investigating the movement of fire induced smoke flow through complex geometry enclosures, and indeed salt water flows have been used in the past to verify computational modelling ${ }^{[6][7]}$. The salt water experiments conducted in this research, represent the early stages of fire induced smoke flow within a residential scale building ${ }^{[8]}$.

In the salt water experiments Laser Induced dye Fluorescence (LIF) has been used to measure the fluid density and to visualise the saline flow in a single vertical plane. The LES and DNS techniques in the FDS have been used to simulate the salt water experiments. The computational predictions of the model have been compared with the experimental measurements of fluid density. The results of the research provide a degree of verification of the FDS hydrodynamic model, in simulating inert buoyant Boussinesq flows within a series of quiescent environment enclosures. The uncertainty of the experimental data has been quantified and is presented in reference [8].

\section{EXPERIMENTAL APPARATUS AND PROCEDURE}

In the experiments, a salt water plume was injected into a two room model where a simple rectangular opening connected the two compartments. As a whole, the model was a rectangular compartment $400 \mathrm{~mm}$ high, $400 \mathrm{~mm}$ wide and $1200 \mathrm{~mm}$ long. This was a scale representation of two ISO fire rooms connected end on end ${ }^{[9]}$. Geometry variations were achieved by changing the opening that connected the two rooms and the compartment opening to the ambient fluid tank. The openings were scaled to represent either a sill (which extended across the full width of the room), or a single-leaf pedestrian doorway. Figure 1shows the four different model geometries used in the experiments.

Salt water was injected into the model through a horizontal square port of $50 \mathrm{~mm}$ internal dimension. The port protruded $67 \mathrm{~mm}$ down from the top of the model and was located 
$300 \mathrm{~mm}$ out from the closed end of the compartment, on the centreline of the model. To distribute the injected saline flow over the entire area of the square port, the rectangular chamber above was packed with porous polyurethane foam and four layers of a fine stainless steel mesh were fixed across the port opening.

LIF was used to illuminate the centreline plane of the saline flow ${ }^{[10][11]}$. The density of the fluid within this vertical plane was measured from the fluorescence emitted by the tracer dye in the salt water. This technique provided an unobtrusive means of collecting continuous fluid density measurements from a single vertical plane in the flow. For comparison with FDS, the time averaged fluid density profiles were collected along line segments that were contained within this centreline plane. Once quasi steady state conditions had been reached, one hundred second time-averaged measurements of the fluid density were taken in both compartments. Within the source compartment eight density profiles were collected over the height of the salt water plume before it plunged into the saline layer. No measurements could be made within the source compartment saline layer, due to problems with refraction. In the compartment that was adjacent to the room containing the salt water plume another eight density profiles were also collected. The fluid density measurements are presented as normalised density difference profiles, where the density difference along the line segment has been normalised by the density difference at the source of the flow. A Cartesian coordinate system, tied to the model geometry, is used to describe the location of the density profiles within the flow field.

Eight different experiments were conducted by using two different salt water densities at the plume source, for each of the four different model geometries. The properties of the saline solution at the plume source and the geometry of the model for each of the eight experiments are detailed in Table 1 . The symbol $\beta$, in Table 1 is a measure of the source buoyancy, as defined by Equation (1). The four different model geometries listed in Table 1 are shown in Figure 1.

$$
\beta=\frac{\rho_{s}-\rho_{\infty}}{\rho_{s}}
$$

\begin{tabular}{|c|c|c|c|c|c|}
\hline $\begin{array}{l}\text { Exp } \\
\text { Title }\end{array}$ & $\begin{array}{c}\rho_{s} \\
\left(\mathrm{~kg} / \mathrm{m}^{3}\right)\end{array}$ & $\begin{array}{c}\rho_{\infty} \\
\left(\mathrm{kg} / \mathrm{m}^{3}\right)\end{array}$ & $\begin{array}{l}\beta \\
-\end{array}$ & $\begin{array}{c}\dot{V}_{s} \\
\left(\mathrm{~m}^{3} / \mathrm{s} \times 10^{4}\right)\end{array}$ & $\begin{array}{c}\text { Geometry } \\
\text { Opening\#1/ } \\
\text { Opening\#2 }\end{array}$ \\
\hline$\overline{\mathrm{CO1}}$ & 1008.88 & 997.96 & 0.01 & 1.023 & Sill / - \\
\hline $\mathrm{CO2}$ & 1019.49 & 999.05 & 0.02 & 1.023 & Sill / - \\
\hline $\mathrm{CO3}$ & 1009.30 & 999.04 & 0.01 & 1.045 & Door / - \\
\hline C04 & 1008.75 & 998.90 & 0.01 & 1.031 & Door / Sill \\
\hline $\mathrm{C} 05$ & 1009.34 & 999.03 & 0.01 & 1.016 & Door / Door \\
\hline $\mathrm{C06}$ & 1019.57 & 998.02 & 0.02 & 1.034 & Door / - \\
\hline C07 & 1019.57 & 998.02 & 0.02 & 1.061 & Door/Sill \\
\hline $\mathrm{C} 08$ & 1019.35 & 997.99 & 0.02 & 1.013 & Door / Door \\
\hline
\end{tabular}

Table 1 Model geometry and plume source conditions for the eight different salt water experiments. 


\section{COMPUTATIONAL MODELLING}

Simulations were conducted to assess the accuracy of the LES and DNS techniques as a function of the uniform grid resolution. It is important to note however, that DNS is normally only employed when the grid cell size is on the order of a millimetre. DNS computations have been conducted in this research because the salt water modelling domain size is relatively small, and knowledge is desired on the accuracy of the DNS technique at crude uniform grid resolutions. Within this paper the uniform grid resolution is described by the parameter KBAR, which equates to the number of grid cells in the vertical dimension of the domain. Thus, the characteristic dimension of the computational grid can be calculated as the domain height divided by KBAR, see Equation 2.

$$
K B A R=\frac{H}{l}
$$

The LES technique employs the Smagorinky sub-grid scale (SGS) model, where the viscosity of the fluid varies spatially in proportion to the local strain rate. The DNS technique, on the other hand, uses a constant viscosity. Computational stability for the DNS technique however requires an artificially high fluid viscosity to dissipate the excess kinetic energy that is passed down to the sub-grid scale from the resolvable large-scale structures. As the resolvable eddy size is dependent on the grid resolution, the minimum fluid viscosity (for computational stability) is also dependent on the grid resolution. The viscosity used in the DNS computations was determined from the grid resolution, in accordance with Equation 3, which was originally derived from DNS simulations of a salt water transitional flow ${ }^{[8]}$.

$$
\mu_{\text {min }}=0.52 \frac{\rho_{\infty} l^{2}}{\sqrt{H}} \sqrt{\frac{\Delta \rho}{\rho_{\infty}} g}
$$

In simulations that employed the default LES technique, the rate of diffusion was specified via the Schmidt number. Although the real Schmidt number for the molecular diffusion of salt in freshwater is of the order of 720, a value of 0.7 was specified in the simulations to maintain computational stability. Thus, the simulations effectively have an elevated rate of molecular diffusion of salt mass in the fluid. The enhanced molecular diffusion could be considered as sub-grid scale modelling of the mass distribution that occurs via small-scale flow structures, which cannot be resolved with the computational grid. In reality however, the turbulent nature of the flow means that the difference in the rate of molecular diffusion (between $\mathrm{Sc}=720$ and $\mathrm{Sc}=0.7$ ) is generally considered to have a negligible impact. In the simulations that used the DNS technique, specifying a diffusion coefficient of $1.581 \mathrm{~m}^{2} / \mathrm{s}$ provided the equivalent rate of diffusion as was used in the LES computations.

The symmetry of the salt water models was not utilised in the simulations to reduce the domain size, as the experimental measurements were collected from the plane of symmetry. Computations were conducted using isothermal conditions where the fluid was incompressible. The fluid densities and viscosities (a lower bound viscosity for LES computations) was specified in the input file. The physical size of the domain and the memory capacity of the computational hardware limited the range of grid resolutions that were assessed. 
Five different uniform grid resolutions are used to conduct a preliminary assessment of the accuracy in simulating the source plume. These resolutions are described by KBAR= 40, 50, 64, 72 and 80. Following on from these simulations, the entirety of each experiment is then simulated twice at a grid resolution defined by $\mathrm{KBAR}=50$, once using the default LES technique and once using the DNS technique. To assess the grid dependence of these simulations, one experiment (exp C04) was simulated at four different grid resolutions described by $\mathrm{KBAR}=20,40,50$ and 62 .

\section{RESULTS}

All eight salt water experiments were simulated using both the LES and the DNS techniques. The size limitation of the paper prevents all of the results being displayed. The complete set of results can be found in reference [8].

The results of the preliminary assessments of how well the salt water source plume is simulated are shown in Figure 2 and Figure 3. Both figures compare the measured density profiles, across the plume with the computational results for five different grid resolutions $(\mathrm{KBAR}=40,50,64,72$ and 80) at eight different elevations within the plume. Figure 2 shows the comparison for the LES technique, while Figure 3 shows the same comparison for DNS. The locations of the density profiles are shown in the legend included in the Figures 3 and 4.

Figure 4 shows a comparison of experimental density profiles and computational predictions at eight different locations downstream, in the compartment adjacent to the source room. The comparison is made for experiment $\mathrm{C} 04$, where the source plume has a buoyancy described by $\beta=0.01$ and the internal geometry of the model has a doorway between the compartments and a sill on the end of the model. The computational results are presented for the LES technique at four different grid resolutions (KBAR= 24, 48, 54 $\& 64)$. The locations of the density profiles are shown in the legend included in Figure 5.

\section{DISCUSSION}

\section{LES versus DNS}

A comparison of the results in both Figure 2 and Figure 3 implies that at crude grid resolutions it is better to use the default LES technique rather than the DNS technique. The DNS computations do not appear to accurately capture the turbulent entrainment in the salt water plume, due to the crude grid resolution and the high fluid viscosity that is required for computational stability. This error, in under-predicting the entrainment in the source plume, carries through into the adjacent compartment, where the buoyancy of the saline layer is then overestimated. The viscosity that is used in the DNS computations has been determined in accordance with Equation (3). It is entirely possible that this empirical relationship simply over-predicts the fluid viscosity, and as such, further research could aim to refine the requirements for stable DNS computations when using the FDS.

As the grid resolution is refined, and more and more flow structures can be resolved by the computations, the viscosity required for computational stability in the DNS technique will approach the real viscosity of the fluids. Thus the performance of the DNS technique can be expected to improve as the grid resolution is refined. In fact, at finer 
grid resolutions it seems reasonable to expect that the DNS technique will perform better than the LES technique. The main reason for this being that the Smagorinsky SGS model can cause artificial fluid behaviour, due to the spatial variation in the fluid viscosity. The point (in terms of grid resolution) where it is preferable to use the LES technique rather than the DNS technique requires further research. If DNS does in fact perform better at finer grid resolutions, this then causes difficulties in choosing a computational technique for simulations where there is a non-uniform grid resolution. In the coarse regions of the grid, the Smagorinsky SGS model may be preferable, while in the fine grid resolution regions (for example, within the ceiling jet) it may be preferable to use DNS. One suspects that the grid resolution in the coarse regions of a non-uniform grid will govern the choice for fire applications.

\section{Dependence on Grid Resolution}

Five different grid resolutions were used in simulating the salt water source plumes. These resolutions equate to the division of the vertical extent of the domain into 40, 50, 64, 72 and 80 uniform grid cells. At these grid resolutions there were 5 to 10 grid cells across the dimension of the plume source. The normalised density difference profiles in Figure 2 and Figure 3 show that the time averaged density profiles across the source plume are relatively insensitive to the resolution of the computational grid in the range $\mathrm{KBAR}=40$ to $\mathrm{KBAR}=80$. The LES computations were more sensitive to the grid resolution than the simulations conducted using the DNS technique. The low sensitivity of the DNS computations to grid resolution, and the over estimation of the peak density difference, is a reflection of the inhibiting effect that the high fluid viscosity (required for computational stability) has on the turbulent mixing occurring in the plume.

The dependence of the computational flow field on grid resolution, for the compartment adjacent to the source room, is illustrated in Figure 4. Within this figure computational results are presented for the LES technique at four different grid resolutions described by $\mathrm{KBAR}=20,40,50,62$. Figure 4 shows that the computational density profiles converge toward the experimental measurements as the grid resolution is refined. Grid resolutions of $\mathrm{KBAR}=20$ and $\mathrm{KBAR}=40$ did not resolve density profiles that clearly showed the presence of the recirculation region downstream of the point where the vent flow plunges into the saline layer. Simulations that had a grid resolution equal to, or greater than, $\mathrm{KBAR}=50$ (which equated to an $8 \mathrm{~mm}$ cell size in the model) generally seemed to resolve the flow features that were observed in experiments. However, there is not a large amount of difference in the time averaged computational density profiles, for the two simulations where $\mathrm{KBAR}=50$ and $\mathrm{KBAR}=62$.

The sharpest interface that was present in the flow field was the density gradient between the saline layer and the ambient freshwater. Accurately resolving the density gradient on the top of the saline layer was one of the greatest sources of disagreement between the computational results and the experimental data. It remains to be seen whether or not simulations with finer grid resolution (KBAR $>62$ ) would converge any further toward the experimental measurement of this density gradient. In the simulation of fire induced smoke flow, this would equate to resolving the interface between the hot smoke layer and the cool lower layer. In the case of the thermal flows however, radiative heating of the ambient layer would assist in smearing out the thermal interface between the flows. Still, care should be taken in considering computational results to take into account that sharp interfaces are not resolved well with numerical methods. 
The results for the DNS computations, in the compartment adjacent to the source room, are not included in this paper. This is because the DNS computational modelling technique did not accurately capture the turbulent entrainment in the source plume at the grid resolutions employed, causing the density of the saline layer in the adjacent compartment to be over estimated significantly. As such, the default LES technique was deemed to be the better approach to employ at the limited grid resolution that was achievable with the computational hardware. All the other experiments were simulated only once, using a resolution of $\mathrm{KBAR}=50$.

\section{CONCLUSIONS}

The research detailed within this paper demonstrates that it is preferable to use the Large Eddy Simulation (LES) technique rather than Direct Numerical Simulation (DNS) at crude grid resolutions, to simulate buoyant saline flows that resemble smoke flow during the early stages of residential building fires. At the uniform grid resolutions used in this work (described by KBAR $<80$ ) the DNS technique did not accurately capture the turbulent entrainment into the source plume because of the artificially high fluid viscosity required for computational stability. In general, the time-averaged computational density results converge toward the experimental measurements, as the grid resolution is refined. LES computations that had a uniform gird resolution with a characteristic cell dimension less than the vertical dimension divided by 50 generally seemed to resolve the flow features that were observed in experiments.

\section{ACKNOWLEDGMENTS}

The authors would like to acknowledge the financial support provided by the New Zealand Fire Service Commission and the Foundation of Research Science and Technology (FRST). Further, we would also like to thank the BFRL at NIST for providing the facilities and personal assistance that supported us in undertaking this research.

\section{NOMENCLATURE}

$$
\begin{array}{ll}
g & \text { - Gravity } \\
H & \text { - Internal height of the compartment } \\
l & \text { - Grid cell dimension (uniform for this paper) } \\
S c & \text { - Schmidt number } \\
\beta & \text { - Source Buoyancy } \\
\rho_{s} & \text { - Saline density } \\
\rho_{\infty} & \text { - Ambient density } \\
\dot{V}_{s} & \text { - Volumetric flowrate of saline at the plume source } \\
\mu_{\text {min }} & \text { - Minimum fluid viscosity required for stable DNS computations }
\end{array}
$$




\section{REFERENCES}

1. McGrattan, K.B. and Forney, G.P. (2000) 'Fire Dynamics Simulator - User's Manual' NISTIR 6469, National Institute of Standards and Technology, U.S. Department of Commerce, Gaithersburg, MD 20899.

2. Baum, H.R. (1999) Large Eddy Simulations of Fires - from Concepts to Computations, Fire Protection Engineering, Issue No 6, pp. 36-42.

3. Steckler K.D., Baum H.R. and Quintiere J.G. (1986) Salt Water Modelling of Fire Induced Flows in Multicompartment Enclosures, NBSIR 86-3327, US Department of Commerce, National Bureau of Standards, Center for Fire Research, Gaithersburg, MD 20899, USA.

4. Fleischmann C. M., Pagni P.J. and Williamson R.B. (1994) Salt Water Modelling of Fire Compartment Gravity Currents, Proceedings of the Fourth International Symposium of Fire Safety Science, pp 253-264.

5. Zukoski E. E. and Peterka D.L. (1979) Measurements of Entrainment in a Doorway Flow, California Institute of Technology, Pasadena, California.

6. Strege, S.M., Quintiere, J. G. and Mitler, H. "Buoyant Plume Dynamics by Salt Water and Large Eddy Simulations", $2^{\text {nd }}$ Joint Meeting of the US Section of the Combustion Institute, 26 March 2001, Oakland, CA.

7. Kelly, A. Qunitiere, J. G. "Examination of Smoke Movement in a Two-Storey Compartment using Salt Water Modelling and Fire Dynamics Simulator" 11-15 June 2001, The International Conference on Engineered Fire Protection Design, SFPE, San Francisco, CA.

8. Clement, J.M. (2000) Experimental Verification of the Fire Dynamics Simulator (FDS) Hydrodynamics Model, Doctoral Thesis, Civil Eng. Dept, University of Canterbury, New Zealand. http://www.civil.canterbury.ac.nz/fire1/rfsresearch.html.

9. International Standard ISO 9705:1993(E). Fire Tests - Full Scale Room Test for Surface Products.

10. Papps, David A. (1995) Merging Buoyant Jets in Stationary and Flowing Ambient Fluids. PhD Thesis, Civil Engineering, University of Canterbury, Christchurch, New Zealand.

11. Gaskin, Susan J. (1995) Single Buoyant Jets in a Crossflow and the Advected Line Thermal. PhD Thesis, Civil Engineering, University of Canterbury, Christchurch, New Zealand. 

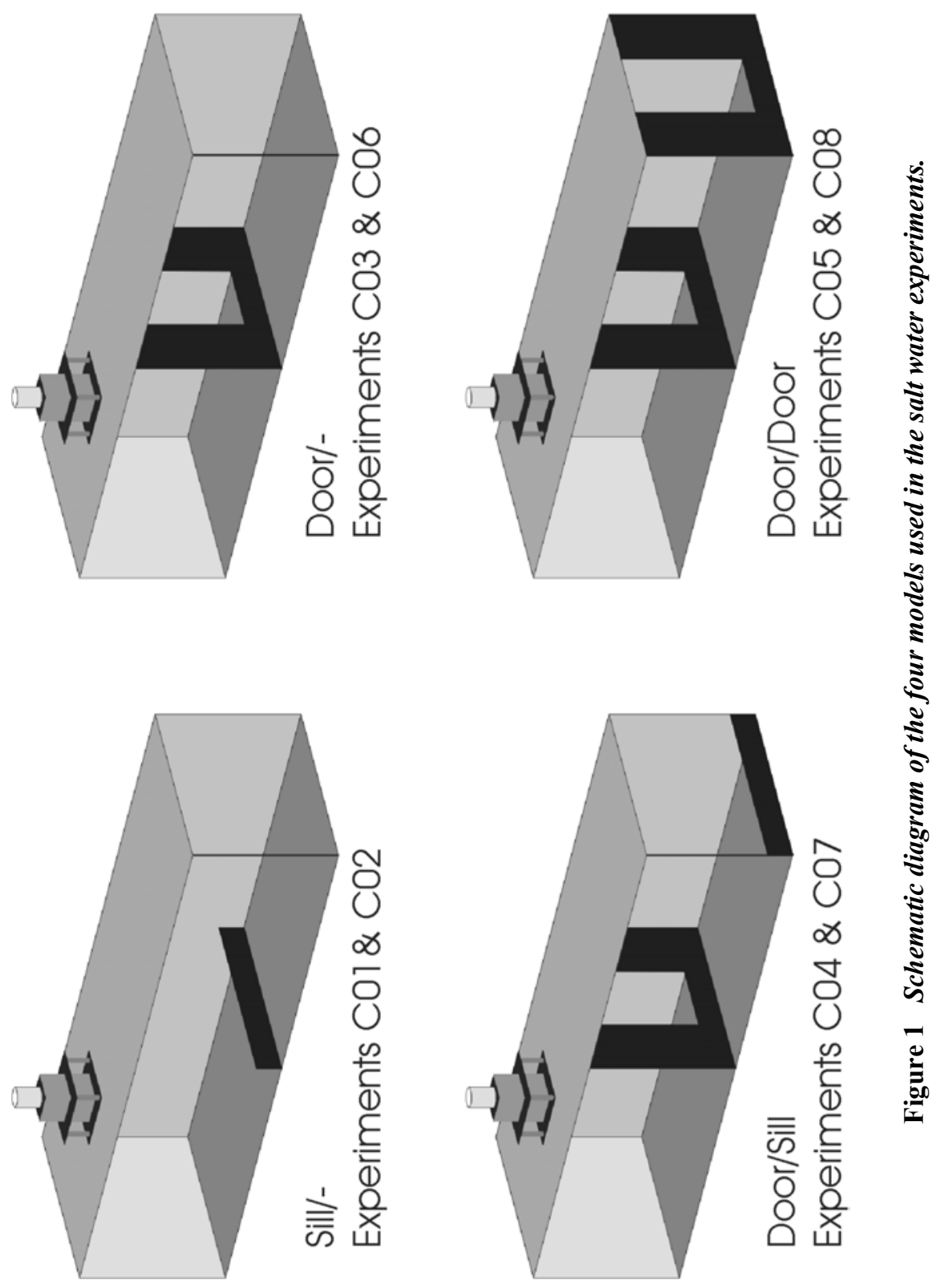

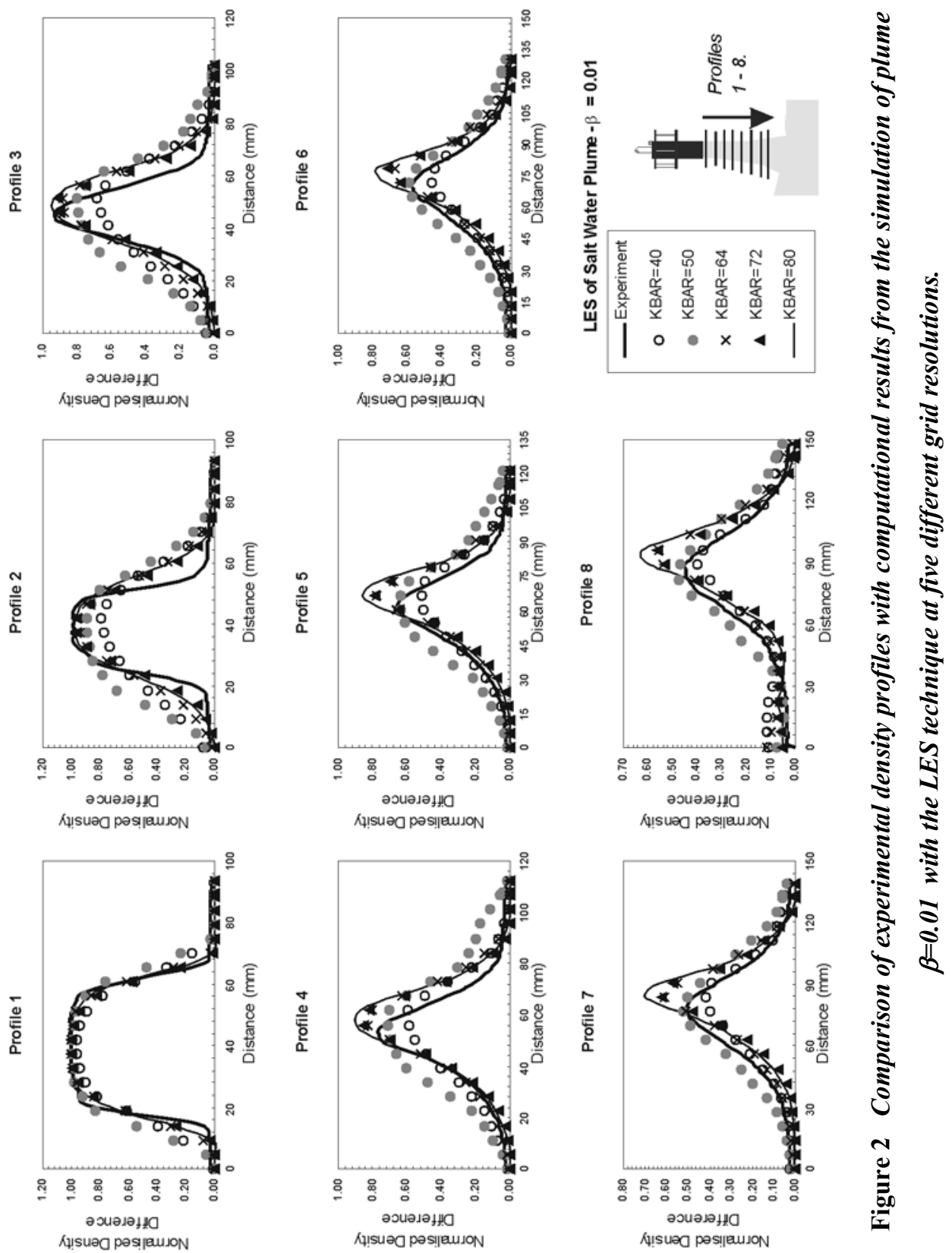


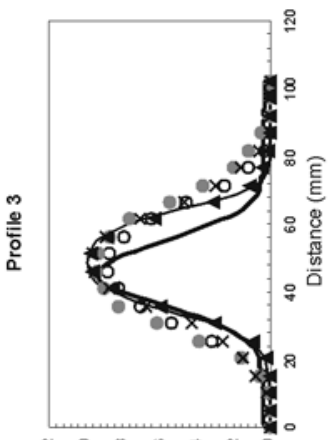

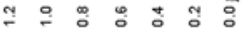

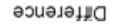

Кџsuea pes!|em.

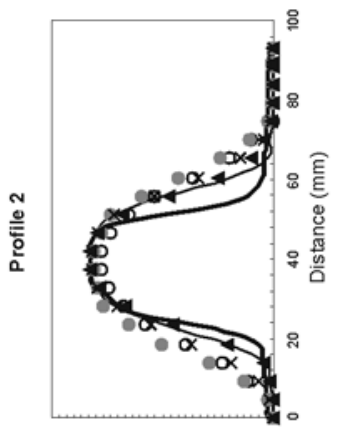

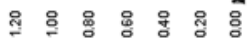

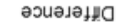

Кsueg pes!iem.

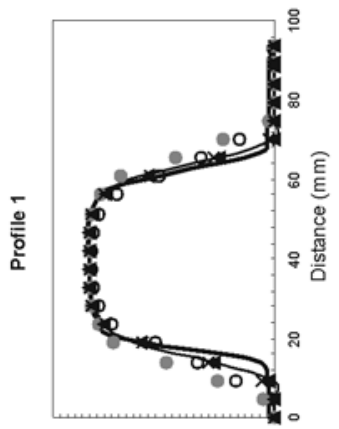

임 웅 융 융 융 정 융

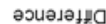

Кџsuəa pəs!|שน,N
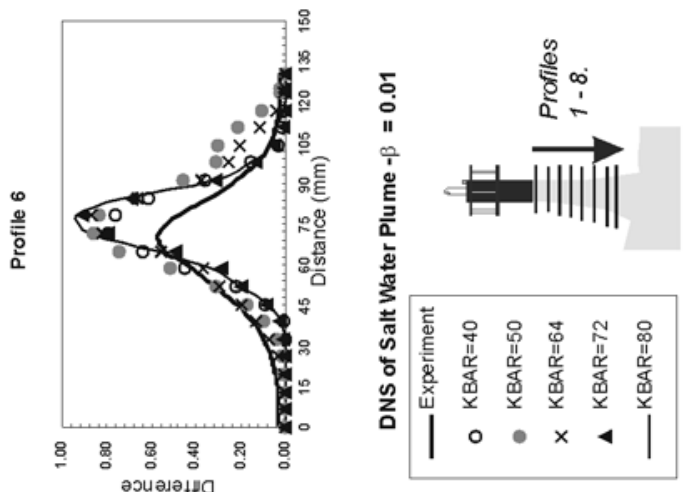

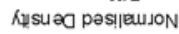
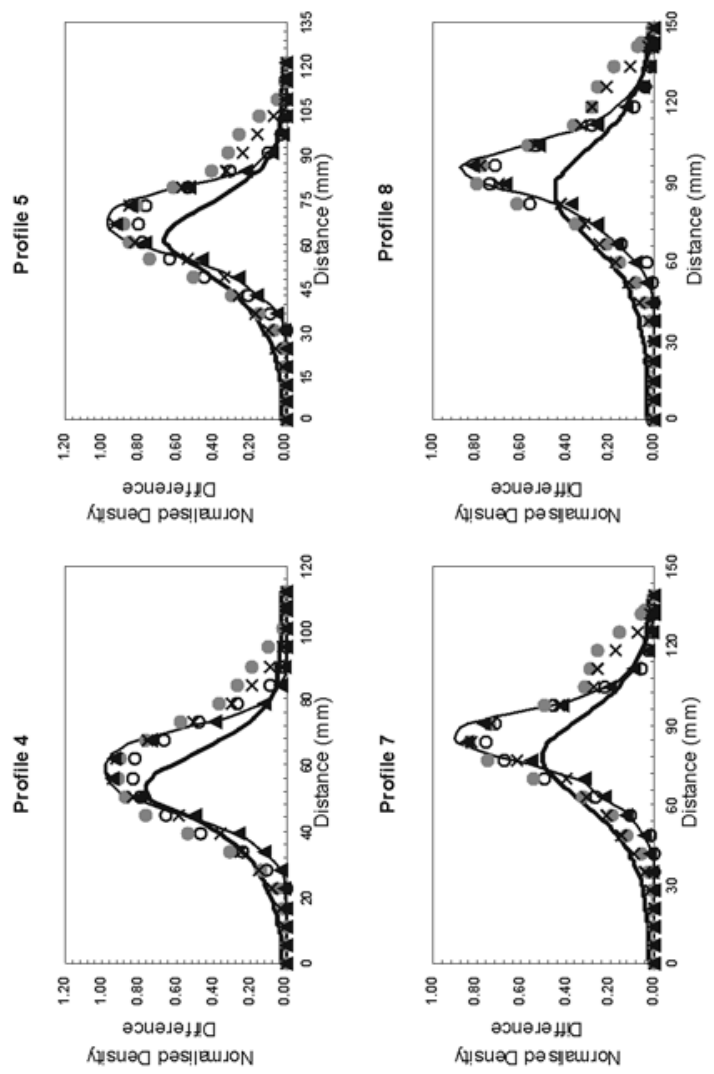

$\underset{5}{5}$

ह

\&

$\pm \frac{2}{3}$

ต

준

의

疍

ถู

ن

\%

\& 9

낭

ㄴ ह

$\geqslant \pm$

ต 2

$\frac{1}{8} \mathbf{Q}$

(1)

ฮั

E

ฮ

잉

प

ธิ

ส

ปิ

() *

$m$

익 $\frac{5}{5}$

으 은 

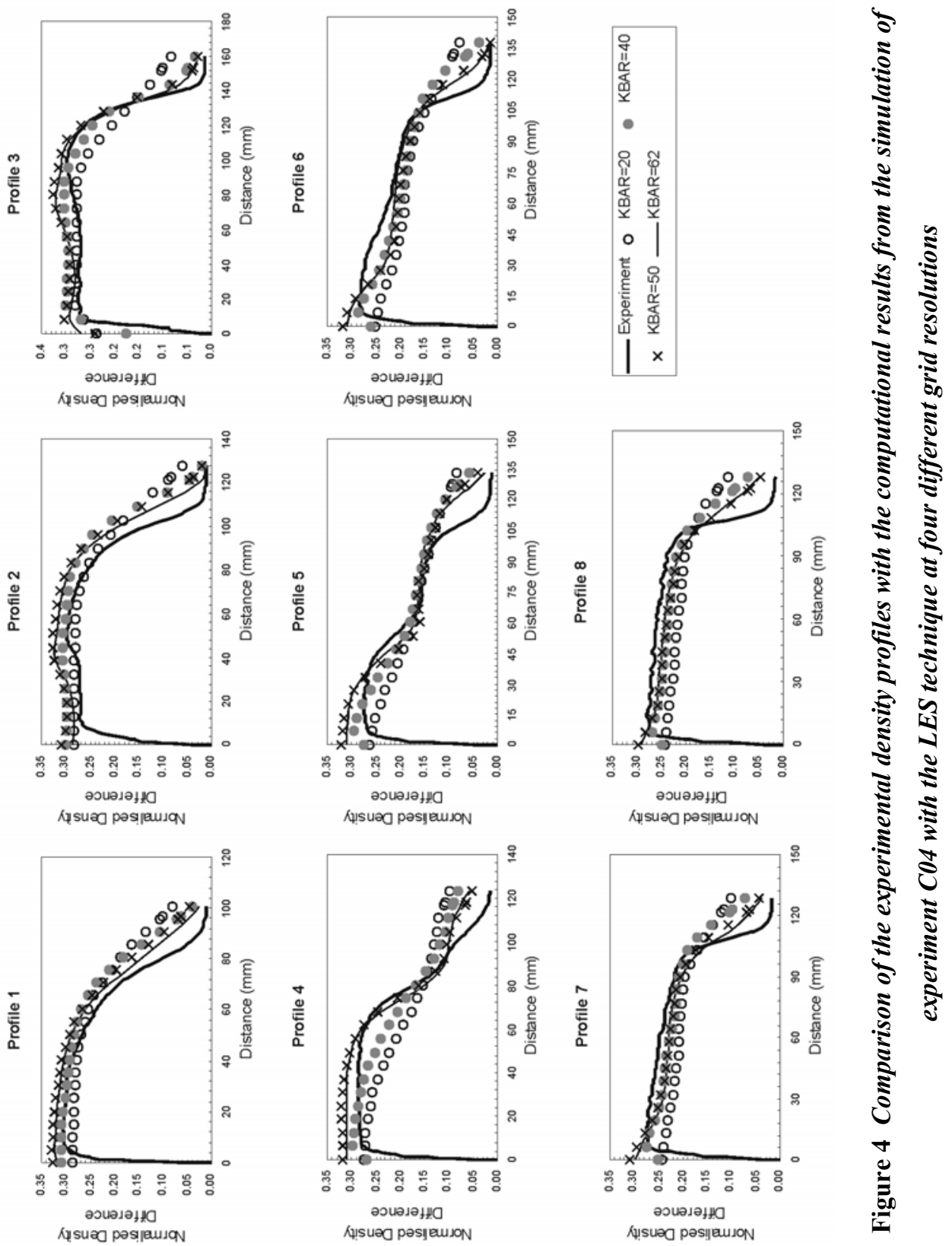